Revista de la Escuela de Ciencias de la Educación. 2022, Año 18 1(17), 78-96. Enero a junio. Baez, M.O.; Ponce de Perino, S.M. y Canossa, C.L. Procesos de alfabetización inicial de jóvenes con síndrome de down: hacia la construcción de una inclusión socioeducativa.

\title{
PROCESOS DE ALFABETIZACIÓN INICIAL DE JÓVENES CON SÍNDROME DE DOWN: HACIA LA CONSTRUCCIÓN DE UNA INCLUSIÓN SOCIOEDUCATIVA
}

INITIAL LITERACY PROCESSES FOR YOUNG PEOPLE WITH DOWN SYNDROME: TOWARDS THE CONSTRUCTION OF A SOCIO-EDUCATIONAL INCLUSION

\author{
Mónica Orfilia Baez \\ Universidad Nacional de Rosario, Argentina \\ monica.baez@unr.edu.ar
}

Stella M. Ponce de Perino

Universidad Nacional de Rosario, Argentina

stellaperino7@hotmail.com

(iD)

\section{Cecilia Lucrecia Canossa}

Universidad Nacional de Rosario, Argentina

ceciliacanossa@gmail.com

Recibido: 29 de julio de 2021

Aprobado: 11 de octubre de 2021

Publicado: 31 de diciembre de 2021

Cita sugerida: Baez, M. O.; Ponce de Perino, S.M. y Canossa, C.S. (2022). Procesos de alfabetización inicial de jóvenes con síndrome de down: hacia la construcción de una inclusión socioeducativa Revista de la Escuela de Ciencias de la Educación. 1(17), 78-96.

\section{RESUMEN}

Este trabajo surge de uno más amplio consistente en un estudio de casos de carácter interdisciplinario con niños y jóvenes con diagnósticos específicos, integrados en escuelas públicas comunes, acerca de cómo aprenden a leer y a escribir con el fin de interpretar las mejores estrategias didácticas $\mathrm{y} / \mathrm{o}$ terapéuticas, para acompañarlos en ese proceso.

En este artículo se informan parcialmente los datos obtenidos a través de una metodología de base psicogenética y sociocultural con tres estudiantes 
Revista de la Escuela de Ciencias de la Educación. 2022, Año 18 1(17), 78-96. Enero a junio. Baez, M.O.; Ponce de Perino, S.M. y Canossa, C.L. Procesos de alfabetización inicial de jóvenes con síndrome de down: hacia la construcción de una inclusión socioeducativa.

adolescentes con Síndrome de Down en situaciones específicas de lectura y de escritura.

La investigación, todavía en curso, pone de manifiesto que los jóvenes indagados desarrollan procesos cognitivos de carácter psicolingüístico complejos y avanzan en el dominio del lenguaje escrito bajo ciertas condiciones didácticas. Estos avances contribuyen a la reflexión acerca de las potencialidades cognitivas de estos estudiantes para el aprendizaje del lenguaje escrito y la incidencia de las modalidades de enseñanza en su posible desarrollo. A partir de este estudio se pretende aportar a la revisión y reformulación de las prácticas educativas en lo que a alfabetización se refiere para lograr la construcción de una educación efectivamente inclusiva.

Palabras clave: Alfabetización - Procesos psicolingüísticos - Educación inclusiva - Síndrome de Down - Prácticas educativas.

\section{ABSTRACT}

This work emerges from a broader one consisting of several interdisciplinary study cases with children and adolescents with specific diagnoses, integrated into common public schools on how they learn to read and write. Therefore, the investigation aims to interpret the best didactic and/or therapeutic strategies to accompany them in that process. Given this background, the present article partially reports the data obtained through a psychogenetic and sociocultural methodology from three adolescent students with Down Syndrome in specific reading and writing situations. The research, still in progress, reveals that the investigated young people develop cognitive processes of complex psycholinguistic character and continue mastering written language under certain didactic conditions. These advances contribute to the analysis of cognitive potentialities of these students for the learning of written language and the incidence of teaching modalities in their possible development. It is intended from this study to contribute to the revision and reformulation of educational practices in terms of literacy to achieve the construction of effective inclusive education.

Keywords: Literacy - Psycholinguistics - Inclusive education - Teacher training - Educational practice.

\section{INTRODUCCIÓN}

En la actualidad se reconoce y se celebra la diversidad de modos de ser y/o de estar desde diferentes discursos disciplinares, educativos o políticos. Particularmente en el ámbito educativo latinoamericano se ha ido gestando un cuerpo normativo que instala, aunque con algunas diferencias regionales, diferentes estrategias educativas para la integración, con miras a su inclusión y a su aceptación, de estudiantes con necesidades educativas especiales. Sin embargo, la sola presencia de estos niños junto a otros, sin diagnósticos específicos, en los mismos contextos escolares no garantiza ni para unos ni para otros la posibilidad de desarrollar los aprendizajes indispensables para participar 
Revista de la Escuela de Ciencias de la Educación. 2022, Año 18 1(17), 78-96. Enero a junio. Baez, M.O.; Ponce de Perino, S.M. y Canossa, C.L. Procesos de alfabetización inicial de jóvenes con síndrome de down: hacia la construcción de una inclusión socioeducativa.

autónomamente en un mundo letrado. La reconfiguración del aula a partir de esta nueva diversidad vuelve dramáticamente visible las áreas de vacancia en la formación de los docentes e incluso en la de profesionales vinculados a la educación, particularmente cuando se trata de la enseñanza de la lectura y de la escritura. Muchos niños y jóvenes son y siguen siendo sujetos de mecanismos de exclusión escolar al comienzo de la escolaridad primaria a partir de su alfabetización. Discriminación que se manifiesta de manera explícita a través de sucesivos fracasos escolares que conducen a la repetición y a la deserción o bien, de manera implícita, posibilitando la permanencia de estos estudiantes en el sistema educativo, aunque no hayan alcanzado los aprendizajes mínimos necesarios que justifiquen sus avances en los distintos niveles del sistema ni dispongan de herramientas para lograr su autonomía y un rol social activo. Este último fenómeno es una forma de exclusión encubierta alentada por las mejores intenciones tal vez pero que evidencia la imposibilidad de interpretar las diferencias y singularidades de los modos de saber y de aprender. La baja calidad en los aprendizajes especialmente en el dominio de la lectura y de la escritura promueve la dependencia y el aislamiento sociocultural, así como el incremento de población en vulnerabilidad social y pobreza extrema. La formación docente y las prácticas de profesionales vinculados al accionar educativo de la escuela (fonoaudiólogos, psicopedagogos, psicólogos) inciden de manera decisiva en esos procesos (Baez y D'Ottavio, 2019).

En el ámbito de la enseñanza y el aprendizaje del lenguaje escrito, error y corrección son términos estrechamente ligados en las prácticas de docentes y de estudiantes, ya que se privilegia la función de control de la institución escolar sobre la base de normas - de "lo normal"- naturalizadas. Las particularidades y lógicas internas de las conceptualizaciones de los estudiantes han sido a menudo soslayadas por priorizar la necesidad de evaluarlos. Tal vez por ello, los "errores" adquieren otra dimensión, si se quiere más dramática, pues no sólo preocupan a los docentes, sino que algunos de estos fenómenos son interpretados desde la perspectiva de la clínica de base neurobiologicista como invalidantes para cualquier proceso de aprendizaje. Por lo que, según cómo interprete el docente o el profesional del lenguaje el valor del error-como carencia o como modo de saber-, el sujeto de aprendizaje podría sumar a su condición de alumno la categoría de "problema", de repetidor, desertor y/o paciente.

En este sentido, el estudio del que se da cuenta parcialmente en este trabajo surge de la necesidad de contribuir a las prácticas de enseñanza, a la formación docente y a la de otros profesionales vinculados a esta problemática (psicopedagogos, fonoaudiólogos, psicólogos, etc.) a partir de la interpretación de los procesos intelectuales que desarrollan niños y jóvenes muchas veces excluidos de antemano de la posibilidad de aprender. Dicha indagación procura específicamente obtener datos para dar sentido a los conocimientos y modos de construcción cognoscitiva de niños y jóvenes con experiencias escolares en escuelas comunes $y / 0$ especiales en las que permanecen durante mucho tiempo y que, sin embargo, no evidencian haber alcanzado el dominio del lenguaje escrito, lo que les impide el acceso a otros conocimientos de importancia escolar y social. Esa permanencia en un "no saber" en los ámbitos escolares obliga a 
Revista de la Escuela de Ciencias de la Educación. 2022, Año 18 1(17), 78-96. Enero a junio. Baez, M.O.; Ponce de Perino, S.M. y Canossa, C.L. Procesos de alfabetización inicial de jóvenes con síndrome de down: hacia la construcción de una inclusión socioeducativa.

comprender los procesos de aprendizaje acerca de la escritura y de la lectura de la que esos sujetos son capaces. A la vez, la reflexión acerca del rol de las intervenciones docentes (las del docente del aula y las de su docente integrador) en esos procesos se vuelve inevitable.

Si se atiende a las prácticas docentes que han caracterizado el aprendizaje de la lectura y la escritura desde una perspectiva tradicional, vinculada al rol más conservador de la institución escolar, algunas de esas prácticas se han consolidado como centrales para el dominio de este campo, situaciones didácticas que se proponen fuera de todo contexto significativo y de toda situación comunicativa, dando por sentado que los estudiantes no disponen de saberes previos, aunque estos sean no convencionales y que el objeto de enseñanza es transparente e inmutable. En este sentido,

(...) hay prácticas que llevan a que lo que hay que conocer esté dado de una vez por todas, como un conjunto cerrado, sagrado e inmutable de cosas transmisibles, pero no modificables. Hay prácticas que llevan a que el sujeto (...) quede fuera del conocimiento, como espectador pasivo o receptor mecánico, sin encontrar nunca respuestas a los por qué y a los para qué que ya ni siquiera se atreve a formular en voz alta (Ferreiro, 1984, p.160).

Es así que para ciertos estudiantes el aprendizaje del lenguaje escrito es un camino lleno de obstáculos, tal es el caso de las minorías lingüísticas y culturales y de los sujetos con necesidades educativas especiales.

\section{DESARROLLO}

\section{De etiquetas y pronósticos}

En América Latina, todos los países han suscrito la Declaración de Salamanca promovida por UNESCO (1994) para la inclusión escolar de personas con necesidades educativas especiales en las escuelas comunes. También son muchos los países, entre ellos Argentina, que han adherido a la Convención sobre los Derechos de las Personas con Discapacidad (Ley No 26378/08) que desde una perspectiva más amplia valida a la inclusión educativa como un Derecho Humano inalienable. Sin embargo, esta temática se vuelve definitivamente visible y normativa en los ámbitos escolares y en las políticas educativas de nuestro país a partir de la Ley de Educación Nacional no. 26206 sancionada en el 2006. Allí la Educación Especial es reconocida como una modalidad del Sistema Educativo, con un carácter de obligatoriedad incluyendo el nivel secundario. Esta ley constituye el molde legislativo de un nuevo paradigma educativo basado en los conceptos de integración e inclusión.

La presencia de estudiantes tradicionalmente ausentes en las aulas ha promovido experiencias de integración en algunos casos emocionalmente interesantes, pero no siempre valiosas respecto del dominio de saberes escolar y socialmente relevantes. Así, observamos con frecuencia que se generan espacios estancos en una misma aula, surgiendo nuevas etiquetas estigmatizantes: los "integrados" y los "comunes o normales". Ese aislamiento- producto de prejuicios 
Revista de la Escuela de Ciencias de la Educación. 2022, Año 18 1(17), 78-96. Enero a junio. Baez, M.O.; Ponce de Perino, S.M. y Canossa, C.L. Procesos de alfabetización inicial de jóvenes con síndrome de down: hacia la construcción de una inclusión socioeducativa.

y preconceptos no teorizados-, tiene como consecuencia que para esos niños las posibilidades de aprendizaje en los ámbitos escolares sean escasas.

La lógica del etiquetamiento, aun reconociendo y proclamando la tolerancia hacia todos, encubre las interacciones, las tensiones, los préstamos, las negociaciones, los saberes y los conflictos que caracterizan especialmente a las relaciones marcadas por las diferencias que han remitido tradicionalmente a la deficiencia.

¿Qué sucede cuando un niño posee un diagnóstico? ¿Es posible ver algo de esas construcciones intelectuales constatadas en muchos niños en el proceso de alfabetización? ¿Qué ocurre entonces cuando lo diferente irrumpe, y el "control" de lo que sucederá en adelante se desdibuja?

La comprensión de los procesos que desarrollan jóvenes y niños estigmatizados como "otros" no solo los reivindica en su singularidad sino también permite interpretar el sentido del "nosotros" que los excluye.

La estigmatización de ciertos sujetos en los espacios educativos se engarza con la que, en las prácticas sociales, sustenta a los prejuicios. Al respecto, como sostiene Castorina (2011):

los daños o deficiencias son nombres para propiedades naturales, las que en su dependencia del contexto llegan a ser limitaciones funcionales para las actividades de aprendizaje. Siempre el daño (debilidad mental, sordera o ceguera, PC, etc.) involucra elementos físicos, una condición que es indeseable respecto de un funcionamiento orgánico o social. Sin embargo, la discapacidad propiamente dicha es un fenómeno relacional que consiste en una relación entre un fenómeno natural y el mundo cultural circundante. Se trata, inevitablemente, de un constructo social: lo que distingue a la discapacidad de la deficiencia es que supone estructuras y mecanismos que no pueden ser reducidos a las características biológicas de los individuos, pero no puede ser reducida a puro 'discurso' como en el constructivismo social (p.30).

Los (pre)juicios en tanto constructos históricos y sociales, obturan la posibilidad de interpretar y dar sentido a la complejidad e historicidad de las trayectorias sociales e individuales, es decir, de las prácticas y de los individuos que las encarnan. Atendiendo a las características de la población con la que se ha trabajado en el estudio al que se refiere esta exposición, es pertinente sostener con Castorina (2011):

Sin duda, el diagnóstico de la trisomia 21 (síndrome de Down) tiene una historia social, con consecuencias para las vidas de los afectados. Sin embargo, más allá de que la trisomia 21 es una construcción social científica, es un término para un hecho natural. Con independencia de cualquier construcción social, alguien tiene o no tiene una trisomia 21. En otras palabras, mientras la "diagnosis del Síndrome de Down" es una construcción social, la trisomía 21 es un hecho natural. Sin duda hay correlaciones estadísticas entre este hecho biológico y otros hechos biológicos. Pero este hecho involucra consecuencias que son sociales, el Síndrome de Down es una categoría social (p. 30).

En igual sentido, Lentini (2008) señala el peso del discurso médico- 
Revista de la Escuela de Ciencias de la Educación. 2022, Año 18 1(17), 78-96. Enero a junio. Baez, M.O.; Ponce de Perino, S.M. y Canossa, C.L. Procesos de alfabetización inicial de jóvenes con síndrome de down: hacia la construcción de una inclusión socioeducativa.

psiquiátrico en la construcción de la categoría "discapacidad mental":

El discurso médico-psiquiátrico desemboca en una construcción objetalizante de la discapacidad mental: su mirada se dirige al déficit, al trastorno, a la disfunción, y en ese mismo movimiento difumina los aspectos singulares y subjetivos del individuo hasta fundirlos en la patología. No trata sobre personas con discapacidad mental: trata, desde un enfoque esencialista, sobre idiotas, imbéciles y débiles mentales, cuya identidad se halla forjada por el síndrome, por la presencia masiva de la anormalidad. En este sentido, la posición del saber psiquiátrico es indisociable de la producción de un orden que plasma en la cuadrícula social el lugar asignado a la discapacidad mental, predicando de este modo su gestión y regulación mediante dispositivos institucionales (p.456).

La atribución de esta categoría a un sujeto genera ciertas expectativas y pronósticos acerca de sus posibilidades educativas, entre ellas respecto de la alfabetización. Sabemos que existen sujetos con este diagnóstico específico que se alfabetizan y una amplia bibliografía da cuenta de sugerencias didácticas al respecto. Sin embargo, la pregunta que nos orienta es indagar las características de los procesos psicolingüísticos que subyacen al logro de ese conocimiento a fin de reinstalar al sujeto, como a cualquier sujeto, en sus propios aprendizajes y contribuir al diseño de situaciones educativas y/o didácticas que los tengan en cuenta.

Es por esto que el objetivo de este artículo es compartir algunos datos que revelan la singularidad y congruencia de los procesos desarrollados por los jóvenes con Síndrome de Down indagados respecto de los procesos ya constatados en niños (Ferreiro y Teberosky, 1979) y adultos (Ferreiro, 1994, 2007) sin diagnóstico previo, a fin de contribuir a la revisión de las situaciones de intervención educativa de la que suelen ser partícipes en condición de integrados o no. Este interés se funda en la convicción de que la comprensión de los procesos de quien aprende orienta estrategias de enseñanza de calidad y, sobre esta base, en la necesidad de aportar a la construcción de ámbitos educativos y escolares efectivamente inclusivos. En este sentido, los objetivos a largo plazo de la indagación que se lleva a cabo son:

- Aportar al análisis e interpretación crítica de los modos de organización y dinámicas vigentes en los procesos de integración escolar.

- Revisar, problematizar y eventualmente reformular parámetros teóricos vigentes en las prácticas educativas y terapéuticas relativas a la alfabetización de sujetos con diagnósticos específicos.

- Explorar y describir los procesos de alfabetización que desarrollan los sujetos seleccionados a partir de las intervenciones propuestas.

En síntesis, el presente artículo pretende contribuir a una reflexión pedagógica y terapéutica a partir de la información parcial de los datos obtenidos hasta el momento que nos permiten sostener que los sujetos que integran nuestra muestra también piensan acerca de la escritura. 
Revista de la Escuela de Ciencias de la Educación. 2022, Año 18 1(17), 78-96. Enero a junio. Baez, M.O.; Ponce de Perino, S.M. y Canossa, C.L. Procesos de alfabetización inicial de jóvenes con síndrome de down: hacia la construcción de una inclusión socioeducativa.

\section{Metodología}

El marco epistemológico general asumido en esta indagación es el provisto por la epistemología genética (Piaget, 1978; Piaget y García, Ferreiro, 1999, García, 2000, 2006a, 2006b, 2011). Desde este enfoque de carácter evolutivo se estima que:

[...] si todo conocimiento es siempre un devenir que consiste en pasar de un conocimiento menor a un estado más completo y eficaz, resulta claro que de lo que se trata es de conocer dicho devenir y de analizarlo con la mayor exactitud posible. De todas formas, este devenir no tiene lugar al azar, sino que constituye un desarrollo (Piaget, 1985, p.13).

La reconstrucción de ese devenir, que permite determinar cuáles son las relaciones entre un sujeto y un objeto de conocimiento particular, requiere de diferentes estrategias metodológicas que permiten explorar desde la perspectiva de los sujetos sus sistemas de interpretación. Así, en este estudio se plantea como un desafío la indagación de los aciertos -entendidos como modos de "saber"- y no los errores o carencias que se observan en las lecturas y en las producciones escritas de sujetos que ya se encuentran marcados por alguna diferencia o discapacidad, pero que manifiestan claras posibilidades de aprendizaje y, en particular, del lenguaje escrito.

El equipo que lleva adelante esta investigación es de carácter interdisciplinar conformado por fonoaudiólogas, psicólogas, psicolingüista, docentes y estudiantes de la Especialización en Alfabetización e Inclusión (radicada en el Centro de Estudios Interdisciplinarios de la Universidad Nacional de Rosario, Argentina). En este sentido, García (2006b) sostiene:

La diferencia fundamental entre una investigación interdisciplinaria y las llamadas investigaciones multi (o "trans") disciplinarias está en el modo de concebir una problemática y en el común denominador que comparten los miembros de un equipo de investigación [...] Dicho de otra manera, mientras que en un caso lo que se integra son los resultados de diferentes estudios sobre una problemática común, en el caso de la interdisciplina la integración de los diferentes enfoques está en la delimitación de la problemática (p.33).

En orden a esto, dicho equipo se constituyó en una primera etapa centrada en la construcción del objeto de estudio y la definición de la metodología de indagación. En una segunda etapa, aún en curso, se desarrolla el estudio del que informamos parcialmente en este trabajo teniendo en cuenta al método de exploración crítica propuesto por Piaget (Piaget, 1973 y 1993; Castorina et. al., 1997) entendido no sólo como un instrumento para la obtención de datos sino como una perspectiva que habilita dialécticamente la formulación y reformulación de estrategias y problemas.

Primera regla de oro para aplicar esa metodología: estar disponibles para lo imprevisto. La convicción de que el adulto es incapaz de reconstruir su propia génesis intelectual obliga a la prudencia, a no despreciar lo insólito, a estar 
Revista de la Escuela de Ciencias de la Educación. 2022, Año 18 1(17), 78-96. Enero a junio. Baez, M.O.; Ponce de Perino, S.M. y Canossa, C.L. Procesos de alfabetización inicial de jóvenes con síndrome de down: hacia la construcción de una inclusión socioeducativa.

atentos a los menores indicios que nos permitan ingresar a lo desconocido. Es preciso dejarse guiar por niños de cuatro o cinco años, aceptando que son interlocutores válidos (Ferreiro, 1996, p.183).

Sostenemos una perspectiva semejante, pues el propósito de esta investigación es comprender los procesos de los que son capaces y el sentido de los mismos, más allá o más acá del Síndrome, atendiendo a la centralidad constructiva del sujeto que aprende y a la naturaleza histórica y compleja del objeto de conocimiento: la escritura.

De manera coherente con las perspectivas teóricas señaladas hasta aquí para la delimitación de la problemática-la interpretación de los procesos de aprendizaje del lenguaje escrito que llevan a cabo estudiantes adolescentes con Síndrome de Down- se han tenido en cuenta los aportes de las investigaciones psicolingüísticas de base psicogenética sobre la construcción de la naturaleza alfabética de distintos sistemas de escritura desarrolladas en diferentes poblaciones infantiles así como la reconstrucción de diferentes aspectos del lenguaje escrito (Ferreiro y Teberosky, 1979, Ferreiro, 1983, 1984, 1994, 2013, 2018, 2019, entre otros). Se trata del recorte de una realidad compleja y complicada pues en las trayectorias escolares de niños y jóvenes con Síndrome de Down confluyen múltiples factores, algunos de ellos compartidos con niños y jóvenes sin diagnóstico de discapacidad, como factores pedagógicos, didácticos e institucionales que inciden en los procesos de alfabetización de cualquier estudiante.

Se considera que las perspectivas citadas son compatibles con el paradigma de salud en el que también se apoya este trabajo, pues a través del mismo no se trata de corroborar una deficiencia sino de analizar e interpretar la diferencia como posibilidad y disponibilidad de otros conocimientos, otros modos de ser y de aprender.

Sobre las bases de lo antedicho, a través de situaciones específicas de lectura y de escritura no escolares ni escolarizadas y del diálogo con cada joven en base a ellas, se procuran interpretar como saberes los argumentos y producciones que manifiestan a través de las actividades propuestas.

En síntesis y en consonancia con lo antedicho, este estudio consiste en una investigación aplicada de tipo exploratorio y descriptivo de carácter cualitativo y evolutivo. La misma se define también como un diseño de campo que se ajusta a las características de un estudio de casos (Stake, 2007) De modo que las variables son medidas a partir de la aplicación de instrumentos confeccionados a tal efecto, en forma sincrónica y diacrónica a toda la muestra pero analizando minuciosamente las variables históricas, vivenciales y situacionales que juegan en cada sujeto- caso y los procesos que los sujetos y los profesionales intervinientes despliegan en cada contexto de aplicación. En este sentido, esta metodología se ha mostrado pertinente al marco teórico que la fundamenta y a los objetivos de la investigación que se propone.

De los casos abordados, en esta presentación daremos cuenta de los avances obtenidos hasta el momento en la indagación y el seguimiento de tres jóvenes (de 19, 16 y 15 años respectivamente) con diagnóstico de Síndrome de 
Revista de la Escuela de Ciencias de la Educación. 2022, Año 18 1(17), 78-96. Enero a junio. Baez, M.O.; Ponce de Perino, S.M. y Canossa, C.L. Procesos de alfabetización inicial de jóvenes con síndrome de down: hacia la construcción de una inclusión socioeducativa.

Down (SD). Todos estos sujetos asisten tanto a escuelas comunes con tutores integradores de tiempo completo, como a espacios de educación especial de manera complementaria, y desarrollan además actividades en instituciones terapéuticas de la ciudad de Rosario.

En estos jóvenes son evidentes y manifiestos los signos corporales con los que se identifica al Síndrome, al punto que la nominación y la imagen que se asocia alcanzan un alto grado de imbricación, al pronunciar el diagnóstico las características físicas resultan inmediatamente evocadas. Entonces, socialmente estos sujetos dejan de ser nombrados en la singularidad de su nombre propio y pasan a ser "el/la Down".

Las situaciones de intervención se previeron desde una perspectiva psicogenética y sociocultural, es decir procurando proponer situaciones contextualizadas y significantes para los entrevistados, entendiendo que leer y escribir son actividades culturales y lingüísticas y por lo tanto no reductibles a grafías y sonidos aislados. Por el contrario, se interpreta que estos adquieren sentido cuando se vinculan en un texto para la producción de significados. Es decir, desde la perspectiva de este estudio el reconocimiento de la relación grafemas/fonemas es un punto de llegada, no un prerrequisito.

Los encuentros con los jóvenes se desarrollaron en una sala de un Centro Terapéutico al que asisten todos los sujetos indagados, quienes se conocen y mantienen relaciones de amistad entre sí.

A través de entrevistas individuales a las que se les imprimió un carácter lúdico, se propusieron consignas siempre contextualizadas a partir de una diversidad de materiales escritos reales, literarios y cotidianos, desde soportes variados como tarjetas y letras móviles, siempre procurando crear un contexto comunicativo que diera sentido a la consigna a trabajar. En diferentes y sucesivas sesiones se trabajó con cada joven, hasta la fecha, sobre:

a) Identificación y escritura del nombre propio.

b) Identificación y escritura de nombres pertenecientes al grupo

c) Escritura de lista de compras para el supermercado.

d) Lectura de títulos de libros de cuentos e interpretación de paratextos

e) Selección de libros para su posterior lectura a cargo del entrevistador o por sí mismos.

f) Escritura/copia de títulos de libros elegidos.

En cada entrevista, las intervenciones de la investigadora procuraron orientarse a la interpretación de los quehaceres de los sujetos, sin atender a la corrección o no -desde la óptica de un sujeto letrado- de esos procedimientos, y a los sentidos y saberes que podían sustentar los argumentos y realizaciones orales o escritas. Las mismas se evaluaron en términos de "convencionales" o "no convencionales" teniendo como referencia la descripción de los procesos psicogenéticos constatados en niños sin diagnóstico previo (Ferreiro y Teberosky, 1979). Cada sesión de entrevista fue videada, se realizó el registro etnográfico y, se transcribió con posterioridad la interacción dialógica surgida en cada uno de los encuentros. En el comienzo del estudio, las primeras sesiones se orientaron a establecer el punto de partida de cada sujeto a indagar. 
Revista de la Escuela de Ciencias de la Educación. 2022, Año 18 1(17), 78-96. Enero a junio. Baez, M.O.; Ponce de Perino, S.M. y Canossa, C.L. Procesos de alfabetización inicial de jóvenes con síndrome de down: hacia la construcción de una inclusión socioeducativa.

\section{Resultados (preliminares)}

Expondremos de manera parcial algunos resultados porque el estudio aún no ha concluido y solo deseamos plantear aquellos que permitan reflexionar sobre la necesidad de comprender que estos jóvenes piensan - como todos los sujetos, aunque con particularidades que aún no sabemos si atribuir a sus avatares escolares 0 a otras variables implicadas- $y$ esto se estima un aporte para repensar las prácticas escolares. Los tres casos de los que aquí daremos cuenta los identificaremos en este contexto como Lola, Víctor y Sergio para preservar su anonimato. Los tres jóvenes asisten como estudiantes integrados a los primeros años de escuelas secundarias de nuestra ciudad: Rosario, Provincia de Santa Fe, Argentina. $\mathrm{Y}$, por otro lado, portan como asignación social y determinación médica, el diagnóstico de Síndrome de Down.

Estos sujetos provenían de experiencias alfabetizadoras institucionales distintas: Lola, era gran copista, práctica que probablemente obedece a razones de supervivencia como respuesta a exigencias que la escuela les impuso durante mucho tiempo. En su escuela, la biblioteca y los libros ocupaban un lugar "específico" del orden de lo "sacralizado" al que solo se accedía cuando la bibliotecaria lo habilitaba. En cambio, para Víctor, la biblioteca era el salón de clases, con estantes y libros en permanente accesibilidad y disponibilidad. El destino de Sergio fue otro. En la escuela especial a la que asistía y en la que mantenía experiencias de integración parcial con la escuela común -las que en la Provincia de Santa Fe se denominan "de integración compartida"-, en determinado momento desistieron de todo intento de alfabetización de este joven y centraron la propuesta pedagógica en los llamados "talleres de formación laboral y artística". En los tres hay coincidencia respecto de la propuesta alfabetizadora que habían experimentado: se basaba en algunos conceptos básicos de la tradición de enseñanza descontextualizada, basada en la estricta asociación fonema y grafema.

Lola, en cuanto a la lectura, podía deletrear correctamente palabras o enunciados breves indicando el nombre de cada letra, pero le resultaba imposible atribuir algún significado a ese conjunto de grafías, por ejemplo, ante un cartel con la palabra "tomate" (propuesta en una lista para compras en el supermercado) la deletrea correctamente apelando al nombre de las letras y/o al sonido: [te-o /m/a-te]. Cuando se le pregunta qué dice todo junto, se muestra desorientada y dice "no sé". En la lectura de textos se atiene a las ilustraciones. Al proponerle escribir su nombre escribe correctamente su apodo, pero no su nombre completo. Analiza esa escritura de manera global, sin comprender posibles segmentaciones.

Se observa en los procedimientos de esta joven la incidencia de una práctica escolar descontextualizada y basada en el alfabeto como objeto de enseñanza en sí: nombre de las letras, deletreo, fragmentación a la que se suma el hecho de que en algún momento de su extenso recorrido escolar le enseñaron la representación de las letras mediante el sistema dactilológico, pese a que esta joven no es sorda. Por lo que en este punto para Lola leer es recitar el nombre de las letras y escribir es copiar. Por esto, en el comienzo de este estudio podía 
Revista de la Escuela de Ciencias de la Educación. 2022, Año 18 1(17), 78-96. Enero a junio. Baez, M.O.; Ponce de Perino, S.M. y Canossa, C.L. Procesos de alfabetización inicial de jóvenes con síndrome de down: hacia la construcción de una inclusión socioeducativa.

deletrear correctamente, por ejemplo, el nombre propio, los de otros compañeros o los títulos de cuentos, sin poder atribuir algún significado al todo, es decir, leerlos realmente.

A partir de las observaciones antes citadas brevemente, se propusieron las situaciones antedichas, orientadas a interpretar qué hipótesis y actividades desarrollaban en situaciones de escritura y de lectura especialmente diseñadas, como ya se ha señalado.

En una de estas circunstancias se le propuso a Lola seleccionar entre el conjunto de tarjetas con nombres de sus compañeros la que dijera su nombre para colgarlo en el estante de un armario que sería de uso propio. Lola fue seleccionando aquellas tarjetas con nombres que tuvieran alguna letra de su propio nombre. Cuando se le pidió que lo escribiera produjo un procedimiento identificable como de acronimia, porque fue segmentando el comienzo de los nombres elegidos y el final del suyo, uniéndolos en un solo término. Así, escribe ISLAV, resultado de la fragmentación y combinación de Isabella, el nombre original de Sergio, Lola y el nombre original de Víctor (este había sido su última elección y contiene dos letras del nombre real de Lola). Procedimiento lexicográfico y morfológico complejo que constituye un fenómeno frecuente en la lengua para la creación de nuevas palabras. Según Casado:

Por acronimia se entiende aquí el procedimiento morfológico consistente en la formación de una palabra a partir de dos o -muy raramente- tres unidades léxicas, estando representada, al menos una de ellas, por un fragmento (una o más sílabas) de su significante; la primera, por el fragmento inicial de su significante, y la última por el fragmento final del suyo: docudrama (< documental + drama), eurocracia (< europea + burocracia) (1999, citado por Álvarez de Miranda, 2007: s/f).

La definición que aporta la Real Academia Española (2020) permite ampliar a otros fenómenos de segmentación, selección y recombinación de elementos lingüísticos:

Acrónimo De acro- y -ónimo.

1. m. Sigla cuya configuración permite su pronunciación como una palabra; $p$. ej., ovni: objeto volador no identificado; TIC, tecnologías de la información y la comunicación.

2. m. Vocablo formado por la unión de elementos de dos o más palabras, constituido por el principio de la primera y el final de la última, p. ej., ofi(cina) (infor)mática, o, frecuentemente, por otras combinaciones, p. ej., so(und) $\mathrm{n}$ (avigation) a(nd) r(anging) (Real Academia Española, 2020, p. s/p).

Este procedimiento es una de las modalidades de creación de abreviaturas. Al respecto, conviene señalar que estamos tan acostumbrados a las abreviaturas que, como señala Ferreiro (2006) "ya no reparamos en la variedad de modos de composición que las caracterizan. Este tipo de producciones son muy antiguas" $(s / p)$. 
Revista de la Escuela de Ciencias de la Educación. 2022, Año 18 1(17), 78-96. Enero a junio. Baez, M.O.; Ponce de Perino, S.M. y Canossa, C.L. Procesos de alfabetización inicial de jóvenes con síndrome de down: hacia la construcción de una inclusión socioeducativa.

Las abreviaturas de todo tipo proliferaron en la Roma Antigua y también fueron muy empleadas por los copistas de la Edad Media. Se debe tener en cuenta que en ese tiempo los lectores eran pocos y compartían las referencias de los textos, por lo que podían restituir el significado a los vocablos abreviados. Además, si consideramos que las prácticas de escritura están ligadas a las tecnologías y soportes disponibles en cada época y que en tiempos medievales la disponibilidad de superficies de escritura era escasa, por lo que debían utilizarse al máximo cada folio de pergamino, exprimiendo el espacio gráfico lo más posible, lo que justifica la popularidad de las abreviaturas. Señala Ferreiro (2006)

Desde el siglo II anterior a nuestra era (II aC) las inscripciones latinas presentan un abundante empleo de abreviaturas. En general, los nombres propios eran abreviados con las primeras letras $\mathrm{O}$, incluso, con la pura inicial: Aug (Augustus), Ser (Servius), A (Aulus), C (Caius), M (Marcus).

También se abreviaban nombres frecuentes: $f$ (filius, o sea, hijo) y se duplicaba la letra para indicar el plural ff ( $f i l i i$, hijos), un procedimiento que en ciertos usos arcaicos sobrevive aún (por ejemplo, FF.AA. para "fuerzas armadas"). Hay incluso largas expresiones donde todas las palabras están abreviadas con la pura inicial, lo cual supone una comunidad capaz de comprender y restituir lo no escrito (Higounet, 1976).

Analizando estas abreviaturas de la antigüedad romana, Desbordes, F. (1995, p. 204-205) hace el siguiente comentario incisivo, muy pertinente para reflexiones de actualidad:

En realidad, la idea que uno puede hacerse de la escritura varía considerablemente según se la relacione con lo oral anterior que ella debe representar o con lo oral posterior que ella tiene la misión de suscitar. En el esquema [oral1 --> escritura --> oral 2] la escritura se encuentra entre dos funciones: la de ser un análisis de lo oral1, o la de dar los medios para producir lo oral2; ahora bien, se pueden dar esos medios sin pasar por un análisis detallado de lo oral1, y si las consideraciones prácticas se imponen a las consideraciones científicas, el ideal fonográfico ya no tiene sentido (Desbordes, 1955, p. 204-205).

Comentando la enorme profusión de abreviaturas entre los romanos, agrega la misma autora:

(...) las abreviaturas se multiplicaron. Pueden tener una complejidad desconcertante para el profano en ciertos dominios especializados como el derecho (...) tienen su justificación en la idea de que no es necesario detenerse en la representación exacta y minuciosa del sonido para tener una comunicación satisfactoria (por lo menos entre iniciados) (Desbordes, 1955, p.49-50).

Desde el punto de vista de las capacidades de Lola, se observa que realiza un procedimiento notable y original. Recordemos que las investigaciones que sirven de antecedente a nuestro trabajo (Ferreiro y Teberosky, 1979, Ferreiro, 
Revista de la Escuela de Ciencias de la Educación. 2022, Año 18 1(17), 78-96. Enero a junio. Baez, M.O.; Ponce de Perino, S.M. y Canossa, C.L. Procesos de alfabetización inicial de jóvenes con síndrome de down: hacia la construcción de una inclusión socioeducativa.

$1983,1984,1994,2018)$ indican la importancia del nombre propio como primera escritura estable que provee al sujeto un repertorio de grafías identificables como "para escribir", por lo que se ha constatado que para diversas escrituras el alfabetizando apela a las letras de su nombre, aun cuando no pueda escribirlas en la secuencia adecuada al mismo. Lola apela no solo a las grafías de su nombre sino también a la de sus compañeros. Procedimiento que, como se señaló, es congruente con la naturaleza de la lengua escrita. El mismo revela un complejo proceso de selección, segmentación, coordinación y secuenciación. Conviene recordar que los segmentos de nombres que aluden, para Lola, a su propio nombre son los de los jóvenes que integran su propio grupo, que es conocido también por la entrevistadora. Es decir, se trata de un conocimiento compartido.

Esta situación revela además el valor del trabajo con el propio nombre y el de otros, más allá del peso subjetivo que sin duda tiene, como fuente de información para el que se alfabetiza, ya que se trata de escrituras estables. Por lo tanto, se constituyen en repertorio, suerte de "caja de herramientas", de grafías que sin duda "son para escribir".

Es pertinente comentar que, en una situación de lectura en la que se le propuso a Lola la selección de uno entre varios libros elegidos cuidadosamente por los investigadores, de manera consistente con el procedimiento empleado en la situación de escritura, esta joven identifica rápidamente el título del libro elegido. Inmediatamente comienza a leer siguiendo con su dedo dicho enunciado atribuyéndole a cada grafía (letra) el valor de una palabra. Lo destacable es que enlaza el sentido de cada palabra en un enunciado narrativo coherente con el contenido del cuento de referencia, texto conocido gracias a las múltiples ocasiones que se le ofrecen con frecuencia para leer a través de la terapeuta 0 por sí misma, aunque no lo haga convencionalmente. De este modo, en la sesión que se comenta, formula un breve relato, síntesis del cuento elegido, asumiendo la postura enunciativa propia de un narrador marcada por cambios en la entonación de su voz, generando así una suerte de puesta en escena. El procedimiento empleado en la situación de lectura parece congruente con el utilizado en la escritura.

En las sesiones posteriores en las producciones e interpretaciones de Lola se observa la progresiva segmentación de palabras y fragmentos de textos con criterios silábico alfabéticos y finalmente alfabéticos (Ferreiro y Teberosky, 1979). Por ejemplo, logra leer convencionalmente estableciendo las correspondencias convencionales tanto su nombre como el de sus compañeros, asimismo anticipa adecuadamente títulos de textos propuestos, aunque la perturban la segmentación de términos sin sentido pleno, como coordinantes y/o subordinantes (ciertas preposiciones en particular)

Lo antedicho parece sugerir el carácter evolutivo del conocimiento de lo escrito, cuestión ya comprobada en investigaciones precedentes (Ferreiro, 1984, en adelante) que el procedimiento que hemos denominado acronimia, lejos de poner en evidencia un error o una carencia, parece indicar un avance en la comprensión del funcionamiento del sistema de escritura y del lenguaje escrito.

A través de las distintas situaciones que se le fueron proponiendo, esta joven logró no solo identificar su nombre en un conjunto, sino leerlo identificando 
Revista de la Escuela de Ciencias de la Educación. 2022, Año 18 1(17), 78-96. Enero a junio. Baez, M.O.; Ponce de Perino, S.M. y Canossa, C.L. Procesos de alfabetización inicial de jóvenes con síndrome de down: hacia la construcción de una inclusión socioeducativa.

las relaciones parte/todo que supone la escritura. Esta cuestión fue progresivamente corroborando, en sus lecturas y en sus escrituras cada vez más convencionales, la capacidad de esta joven para reconstruir como tantos otros sujetos no diagnosticados la naturaleza de nuestro sistema de escritura.

A la par de situaciones de reflexión sobre la propia escritura o sobre las de otros jóvenes, se realizaron variadas y múltiples situaciones de lectura a partir de la elección de la propia joven de materiales que deseaba que se le leyeran o bien para leerlos por sí misma. Por otra parte, respecto de la actividad de copiado, se fue observando que los avances de Lola en otras situaciones de lectura y de escritura le permitían realizar un trabajo casi autónomo de revisión del texto copiado, identificando errores propios.

Víctor, a diferencia de Lola, al comienzo del estudio manifestaba conocimientos más avanzados, ya que lograba identificar y leer diferentes palabras de manera convencional realizando anticipaciones de significado adecuadas. Recordemos que Víctor es el único de los tres jóvenes indagados para el que el acceso a materiales escrito era cotidiano, lo que pudo incidir en el avance en sus conceptualizaciones. A la hora de escribir, su escritura parecía ajustarse a lo que se conoce como hipótesis silábica (Ferreiro y Teberosky), es decir, a cada grafía que escribía, de modo convencional o no, le atribuía un recorte silábico del término de referencia. Tanto en las situaciones de lectura como de escritura reconocía y producía la segmentación entre palabras de manera convencional, aunque oscilando entre escrituras silábicas o silábico alfabéticas. Su modo de interpretar la escritura se reflejaba también en la lectura de textos, situación en la que también realizaba anticipaciones con las que operaba de manera global. Víctor prefería el trabajo con listas antes que con textos ya que en dicho formato podía controlar mejor su escritura y anticipar la lectura. Sin embargo, a través de cada sesión Víctor manifestó avances notables hacia la escritura y lectura convencional de textos.

Sergio, al igual que los otros jóvenes citados, en el comienzo del estudio diferenciaba dibujo de escritura, era un gran copista y sentía particular fascinación por los libros literarios y sus ilustraciones. Progresivamente y a través de situaciones que capitalizaron su interés por estos materiales escritos fue identificando elementos paratextuales y textuales en situación de lectura, realizando anticipaciones significantes adecuadas, y en sus escrituras llamó la atención su preocupación por atender a aspectos tipográficos. En la escritura del título de su cuento preferido parece apelar al mismo procedimiento empleado por Lola, que hemos llamado de "acronimia". No obstante, esta hipótesis requeriría de más constataciones que no se pudieron realizar pues cuando se tuvo oportunidad de una nueva entrevista Sergio argumentaba desde una hipótesis silábico-alfabética su escritura.

A través de las sucesivas entrevistas, los tres jóvenes manifiestan haber alcanzado el conocimiento de la naturaleza alfabética del sistema y logran leer convencionalmente, aunque aún se están indagando los procesos que desarrollan para dominar todos los dispositivos que hacen a la convencionalidad de los textos escritos. 
Revista de la Escuela de Ciencias de la Educación. 2022, Año 18 1(17), 78-96. Enero a junio. Baez, M.O.; Ponce de Perino, S.M. y Canossa, C.L. Procesos de alfabetización inicial de jóvenes con síndrome de down: hacia la construcción de una inclusión socioeducativa.

\section{CONCLUSIÓN}

Se han observado muchas experiencias de integración escolar que se basan sólo en la buena voluntad de los actores involucrados pero que, por falta de claridad de objetivos, de capacitación, de planificación, de ductilidad o de recursos sabiamente aplicados, fracasan en el caso concreto y ponen en tela de juicio a toda la conceptualización construida en torno a la inclusión escolar.

Son pocas las experiencias de inclusión plena en el Aula Común, basada en la finalidad de una educación en la diversidad. En cambio, están proliferando modelos de integración parcial y limitada, cuyos criterios y principios se basan en diagnósticos clasificatorios y discriminatorios de los niños o jóvenes con discapacidad, enfocados desde el paradigma centrado en el déficit.

Desde este contexto es que se interpreta la necesidad de estudios como el presente, orientados a abordar desde los propios sujetos la elaboración de las estrategias educativas más adecuadas a sus necesidades y a la naturaleza del lenguaje escrito y de las prácticas sociales que le dan sentido.

A lo largo del proceso registrado, estos sujetos evidenciaron diferenciaciones y coordinaciones sucesivas que indicaron la complejidad de las hipótesis que se estaban formulando, así como las transformaciones que operaron respecto de la escritura. Esto nos parece muy relevante, pues se trata de procesos congruentes con los constatados en sujetos que no portan diagnóstico. Este dato, no lo disponíamos al comienzo de nuestro estudio y, precisamente, por el carácter evolutivo evidenciado en los procesos desarrollados por los jóvenes indagados, se cuestionaría el concepto de "plafonamiento", frecuente en el trabajo con sujetos con discapacidad. Además, el tipo de situaciones propuestas, en las que los materiales escritos se presentan en toda su complejidad y posibilidad significante, permitiría interpelar el tradicional paradigma de "didáctica especial diferencial", que fuera en ese momento histórico un concepto importante basado en los trabajos de André Rey (19061965 ) y por el Centro de Investigaciones Foniátricas y Audiológicas que presidía Julio Bernaldo de Quirós (1980) en Argentina.

En este sentido, tradicionalmente, las teorizaciones que acompañaron los diseños curriculares en la escolaridad especial sólo ofertaban contenidos que se correspondieran estrictamente con los "niveles de pensamiento" de los estudiantes, los cuales eran previamente diagnosticados y mensurados con instrumentos objetables. Esas categorizaciones establecidas desde los "cocientes intelectuales" supieron condicionar la vida de más de un sujeto al punto de determinar que no aprendería a leer o a escribir, cuando en realidad no había impedimento demostrado para ello. Se estima, por el contrario, a partir de los datos obtenidos hasta el momento con los sujetos indagados, la posibilidad de pensar, en palabras de Ansermet (2001), en la no-determinación que marca su destino en todos los planos, neurobiológico, genético y social. Es esta nodeterminación lo que le ofrece al sujeto el espacio para realizar sus propias elecciones, apoyándose al mismo tiempo en lo que lo determina. Ya sea en el campo genético, cerebral o social, siempre llegamos a un eslabón perdido, pues el sujeto se halla determinado paradójicamente por la ausencia de su 
Revista de la Escuela de Ciencias de la Educación. 2022, Año 18 1(17), 78-96. Enero a junio. Baez, M.O.; Ponce de Perino, S.M. y Canossa, C.L. Procesos de alfabetización inicial de jóvenes con síndrome de down: hacia la construcción de una inclusión socioeducativa.

determinación. Es así como el sujeto puede ser verdaderamente comprendido como una excepción a lo universal que lo sostiene. Este espacio fundamental de libertad, biológicamente determinado si podemos permitirnos la paradoja, es un factor de imprevisibilidad (p.316).

Al respecto, Filidoro (2010), plantea un modelo de diagnóstico en la clínica, que bien podría revisarse y reformularse para pensarlo en las prácticas educativas que se despliegan en el aula. En este espacio institucional e institucionalizado se parte generalmente de una supuesta ignorancia de los estudiantes, por el contrario, postulamos que disponen de saberes previos que interactúan con los que se proponen en la escuela. En sentido semejante, y subrayando los riesgos del etiquetamiento, la autora citada señala que los datos son observables cuando la producción de un niño es desplegada en el eje diacrónico, por ello no propone una psicopatología nosográfica sino interpretativa. De este modo, Filidoro (2010) sostiene que el niño queda ubicado "en un lugar de imprevisibilidad: el niño no se encuentra anticipado en ningún saber, $[\ldots .$.$] estamos dispuestos a que cada$ niño emerja en un lugar singular, esto es, no previsto (p. 63).

Al respecto, se podría aplicar la afirmación de Madrigal Muñoz (2004) referida a familias de sujetos con Síndrome de Down a la reflexión de los límites y restricciones impuestas por el sistema escolar a estos niños y jóvenes, aún desde las mejores intenciones:

Las familias deben desterrar las creencias que dicen que el desarrollo de las capacidades de los niños y niñas con Síndrome de Down tienen un límite que no puede superar ningún programa de tratamiento ni de aprendizaje. Su evolución es más lenta pero sus potencialidades de desarrollo son mucho mayores que lo que se pensaba hace 25 años ( $p$. 13.)

Publicaciones más actuales, de Fundaciones y Asociaciones de Profesionales y Padres, por ejemplo ASDRA, AISDRO, Fundación Iberoamericana Down21, Fundación Síndrome de Down de Cantabria, entre otras) que trabajan en torno a la educabilidad de los sujetos con Síndromes de Down intentan desarmar ese determinismo, insistiendo en el abordaje más temprano (que ese determinismo demoraba ya que no se lo consideraba necesario) y en la ampliación de los tiempos al momento de pensar en los tiempos de logro.

Precisamente, cuando las potencialidades intelectuales de los estudiantes no se consideran y las correlaciones tiempo y aprendizaje se ignoran, también se desprofesionaliza al docente, postergando su formación como tal y dejándolo carente de herramientas para interpretar la lógica de los quehaceres y haceres de los niños y jóvenes en interacción con este campo de saber cuya reconstrucción conceptual no es sencilla. Para que esto no suceda parece necesario promover la formación de docentes capaces de interpretar positivamente la diversidad del alumnado, de reconocer la complejidad e historicidad de un objeto de enseñanza particular como es el lenguaje escrito, de comprender y aceptar el desafío de explorar la variedad de posibilidades que hoy 
Revista de la Escuela de Ciencias de la Educación. 2022, Año 18 1(17), 78-96. Enero a junio. Baez, M.O.; Ponce de Perino, S.M. y Canossa, C.L. Procesos de alfabetización inicial de jóvenes con síndrome de down: hacia la construcción de una inclusión socioeducativa.

se ofrecen para la alfabetización junto y con sus estudiantes, con diagnóstico o no.

¿Por qué desplazar la formación docente de la educación a la salud procurándoles elementos para "diagnosticar" patologías antes que saberes? ¿Por qué no asegurar la constitución de un rol profesional docente quien, como parte de un equipo interdisciplinario del que participen también profesionales de la salud, pueda asumir la especificidad de su quehacer, es decir, enseñar? ¿Por qué no capacitar a los profesionales de la salud en lo que hoy sabemos acerca de los procesos de aprendizaje? La reflexión sobre las posibles respuestas a estas preguntas, se estima que habilitarán una revisión acerca de la construcción conceptual del rol docente y el de otros profesionales. Además, se estima que quienes reconocen el valor de los conocimientos científicos que se han mencionado debieran asumir el desafío de contribuir a redefinir los paradigmas de normalidad y patología que frecuentemente se aceptan sin cuestionamientos. Esto parece necesario porque ya tenemos elementos para reconocer en el campo de la alfabetización a ese niño/ joven o adulto activo y constructor que la obra de Piaget y la de sus discípulos nos enseñaron a ver. Se estima necesario y urgente instalar las acciones que vuelvan el derecho a la alfabetización una realidad accesible para todos, y con ello la inclusión podría dejar de ser una utopía.

\section{REFERENCIAS}

Asociación Síndrome de Down de la República Argentina (ASDRA) https://www.asdra.org.ar/ Consultado el 8 de septiembre de 2021

Asociación para la Inclusión de las Personas con Síndrome de Down de Rosario (AISDRO) https://aisdro.org.ar/ Consultado el 8 de septiembre de 2021

Baez, M. y D'Ottavio, M.E. (2019). La diversidad en el aula: el desafío de interpretar la singularidad de los procesos de alfabetización inicial. Revista Ciencia y Educación, 3(3).

Bernaldo de Quirós, J. y Schrager, O.L. (1980). Fundamentos neuropsicológicos en las discapacidades de aprendizaje. Editorial Médica Panamericana.

Castorina, et. al. (1997). Psicología Genética. Aspectos Metodológicos e implicancias pedagógicas. Miño y Dávila.

Castorina, J. A. (2011). Problemas filosóficos de la Psicología en la Educación Especial. III Congreso Internacional de Investigación y Práctica Profesional en Psicología XVIII. Jornadas de Investigación Séptimo Encuentro de Investigadores en Psicología del Mercosur.

Ferreiro, E. y Teberosky, A. (1979;1999). Los sistemas de escritura en el desarrollo del niño. Siglo XXI.

Ferreiro, E. (1983). Procesos de adquisición de la lengua escrita dentro del contexto escolar. Revista Latinoamericana de Lectura (Lectura y Vida), $4(2), 11-18$.

Ferreiro, E. (1984). "Los problemas cognitivos involucrados en la construcción de la representación escrita del lenguaje". Actes de les Primeres Jornades 
Revista de la Escuela de Ciencias de la Educación. 2022, Año 18 1(17), 78-96. Enero a junio. Baez, M.O.; Ponce de Perino, S.M. y Canossa, C.L. Procesos de alfabetización inicial de jóvenes con síndrome de down: hacia la construcción de una inclusión socioeducativa.

sobre noves perspectives sobre la representació escrita en el nen, Institut Municipal d'Educació, 159-167

Ferreiro, E. (1994). Apuntes sobre alfabetización, oralidad y escritura. Necesidades educativas de los adultos. Encuentro de especialistas. Instituto Nacional para la Educación de los Adultos, 81-86.

Ferreiro, E. (1996). Aplicar, replicar, recrear. Acerca de las dificultades inherentes a la incorporación de nuevos objetos al cuerpo teórico de la teoría de Piaget. Substatum, 3, 8-9, 175-185.

Ferreiro, E. (1999). Vigencia de Jean Piaget. México. Siglo XXI.

Ferreiro, E. (2006) Nuevas tecnologías y escritura. Docencia, XI(30), 46-53.

Ferreiro, E. (2007). Los adultos no alfabetizados y sus conceptualizaciones del sistema de escritura en E. Ferreiro, E. Alfabetización de niños y adultos. Textos escogidos. Centro de Cooperación Regional para la Educación de Adultos en América Latina y el Caribe (CREFAL).

Ferreiro, E. (2013). El ingreso a la escritura y a las culturas de lo escrito. Siglo XXI.

Ferreiro, E. (2018). Acerca de las dificultades para aceptar que los niños piensan sobre lo escrito. Bellaterra Journal of Teaching \& Learning Language \& Literature (BJTLLL) 11(2).

Ferreiro, E. (2019). Prospectivas en investigación sobre alfabetización inicial. Dicotomías metodológicas y epistemológicas. Infancia y Aprendizaje, $1(42), 1-36$.

Filidoro, N. (2010). Diagnóstico psicopedagógico: los contenidos escolares. La lectura. Editorial Biblos.

Fundación Síndrome de Down de Cantabria. Revista virtual. En http// wwwdowncantabria.com.

Fundación Iberoamericana Down 21. En https://www.down21.org/.

García, R. (2000). El conocimiento en construcción. Gedisa.

García, R. (2006a). Epistemología y Teoría del Conocimiento. Revista Salud Colectiva, 2(2), 113-122.

García, R. (2006b). Sistemas complejos. Conceptos, método y fundamentación epistemológica de la investigación interdisciplinaria. Gedisa.

García, R. (2011). Interdisciplinariedad y sistemas complejos. Revista Latinoamericana de Metodología de las Ciencias Sociales, 1(1). http://www.memoria.fahce.unlp.edu.ar/ .

Lentini, E. (2008). El discurso psiquiátrico sobre la discapacidad mental. XV Jornadas de Investigación y Cuarto Encuentro de Investigadores en Psicología del Mercosur. Facultad de Psicología - Universidad de Buenos Aires.

Madrigal Muñoz, A. (2004). El Síndrome de Down. https://sidinico.usal.es/documentacion/el-sindrome-de-down/

Piaget, J. (1971,1985). Psicología y Epistemología. Planeta Agostini.

Piaget, J. (1973; 1993). La representación del mundo en el niño. Morata.

Piaget, J. (1978). Introducción a la epistemología genética. Paidós.

Piaget J. y Garcia, R. (1982). Psicogénesis e Historia de la Ciencia. Siglo XXI. 
Revista de la Escuela de Ciencias de la Educación. 2022, Año 18 1(17), 78-96. Enero a junio. Baez, M.O.; Ponce de Perino, S.M. y Canossa, C.L. Procesos de alfabetización inicial de jóvenes con síndrome de down: hacia la construcción de una inclusión socioeducativa.

Real Academia Española (s.f.). Diccionario de la Lengua Española. https://www.rae.es/obras-academicas/diccionarios/diccionario-de-lalengua-espanola

Rosato, A. y Angelino, A. (coord.) (2009). Discapacidad e ideología de la normalidad. Desnaturalizar el déficit. Centro de Publicaciones Educativas y Material Didáctico, Noveduc.

Stake, R. E. (2007). Investigación con estudio de casos. Morata

UNESCO (1994). Declaración de Salamanca de principios, política y práctica para las necesidades educativas especiales y marco de acción sobre necesidades educativas especiales. https://unesdoc.unesco.org/ark:/48223/pf0000098427_spa 